\title{
Mechanically and Electrically Robust Self-Assembled Monolayers for Large-Area Tunneling Junctions
}

Yanxi Zhang, ${ }^{\dagger \neq, \perp}$ Xinkai Qiu, ${ }^{\dagger, \ddagger, \perp}$ Pavlo Gordiichuk, ${ }^{\dagger, \perp, \S}$ Saurabh Soni, ${ }^{\dagger \neq}$ Theodorus L. Krijger, ${ }^{\dagger, \ddagger}$ Andreas Herrmann, ${ }^{\ddagger 0}$ and Ryan C. Chiechi* ${ }^{\dagger} \dagger, \ddagger$

${ }^{\dagger}$ Stratingh Institute for Chemistry, University of Groningen, Nijenborgh 4, 9747 AG Groningen, The Netherlands

\#Zernike Institute for Advanced Materials, Nijenborgh 4, 9747 AG Groningen, The Netherlands

Supporting Information

ABSTRACT: This paper examines the relationship between mechanical deformation and the electronic properties of selfassembled monolayers (SAMs) of the oligothiophene 4$\left(\left[2,2^{\prime}: 5^{\prime}, 2^{\prime \prime}: 5^{\prime \prime}, 2^{\prime \prime \prime}\right.\right.$-quaterthiophen $]$-5-yl) butane-1-thiol (T4C4) in tunneling junctions using conductive probe atomic force microscopy (CP-AFM) and eutectic Ga-In (EGaIn). We compared shifts in conductivity, transition voltages of T4C4 with increasing AFM tip loading force to alkanethiolates. While these shifts result from an increasing tilt angle from penetration of the SAM by the AFM tip for the latter, we ascribe them to distortions of the $\pi$ system present in T4C4, which is more mechanically robust than alkanethiolates of comparable length; SAMs comprising T4C4 shows about five
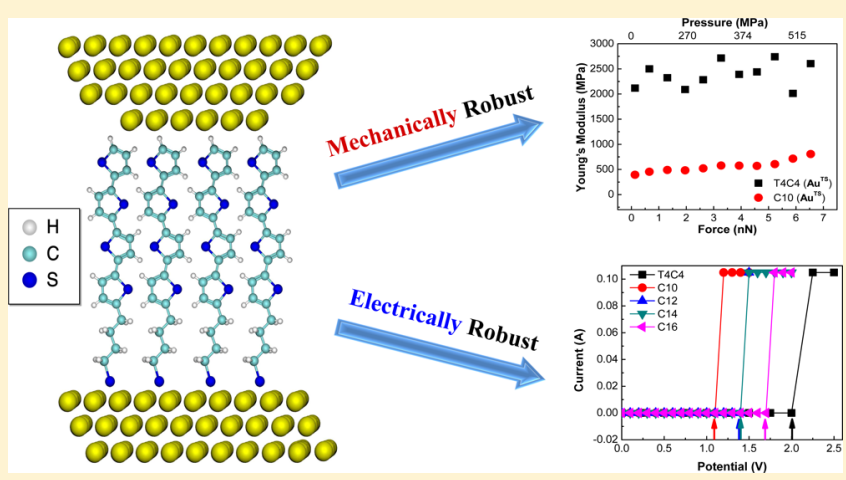
times higher Young's modulus than alkanethiolates. Density functional theory calculations confirm that mechanical deformations shift the barrier height due to changes in the frontier orbitals caused by small rearrangements to the conformation of the quaterthiophene moiety. The mechanical robustness of T4C4 manifests as an increased tolerance to high bias in large-area EGaIn junctions suggesting that electrostatic pressure plays a significant role in the shorting of molecular junctions at high bias.

\section{INTRODUCTION}

Emerging challenges in information technology are driving research into new computer architectures and circuit designs ${ }^{1}$ that require new materials and concepts in nanoelectronics. Molecular electronics, specifically tunneling junctions comprising discrete molecules, are well suited to address these challenges ${ }^{2,3}$ because they control charge-transport directly at the quantum level, however, it remains impractical to integrate single-molecule junctions ${ }^{4,5}$ into devices. Bottom-up junctions comprising self-assembled monolayers ${ }^{6-8}$ (SAMs,) on the other hand, can already be incorporated into wafer-scale fabrication processes ${ }^{9}$ and diode logic circuits. ${ }^{10}$ When molecules pack into a SAM, collective effects, such as the overlap of interacting electronic states and charges, give rise to new properties that affect tunneling charge-transport significantly as compared to single-molecule junctions. ${ }^{11-14}$ In addition to electronic and electrostatic effects, SAMs exhibit mechanical properties derived from the interactions between individual molecules, which play a critical role in the tolerance of SAMs toward particular top-contacts and, ultimately, technological applications. Small changes in the conformation of a molecule or ensemble of molecules (e.g., in a SAM) between two electrodes can have dramatic effects on conductance by altering electronic states in the metal/ molecule/metal junction. ${ }^{.2,15}$ Large-area junctions are typically constructed using SAMs of molecules with anchoring groups such as thiols that drive self-assembly into ordered structures that impose fixed conformations. The effects of these conformations and their relationship to the bulk mechanical properties of the SAM can not be ignored, particularly for $\pi$ conjugated molecules, since intermolecular interactions affect charge transport via electrostatic effects and because both hopping and tunneling charge-transport are sensitive to electronic delocalization, which is maximized in coplanar conformations. Establishing a structure-function relationship between mechanical deformation and electrostatics in SAMs of $\pi$-conjugated molecules is, therefore, important fundamentally and for the potential for exploitation in molecular-scale devices that are sensitive to force/pressure/deformation.

Conductive probe atomic force microscopy (CP-AFM) is capable of characterizing the electrical properties of SAMs while varying the loading force applied to a probe tip that doubles as a top-contact. Changes in the resistances of SAMs of alkanethiolates with applied force have been ascribed to changes in the tilt angle of the alkane chains. ${ }^{16-20}$ Transition voltage spectroscopy (TVS) indicates that the transition voltage $V_{\text {trans }}$ shifts to a lower bias with increasing force (i.e., as the tilt angle increases). ${ }^{19,20}$ This observation implies a decrease in

Received: April 25, 2017

Revised: June 12, 2017

Published: June 13, 2017 
barrier height of molecular junctions because $V_{\text {trans }}$ is proportional to the energy offset between the Fermi level $E_{\mathrm{f}}$ and the highest-occupied molecular state. ${ }^{21}$ If there are more subtle influences to the electrostatics of the junctions from bond distortions they are masked by the larger effect of the tilt angle increasing as the tip penetrates the SAM, which is stabilized only by relatively weak intermolecular dispersion forces. The mechanical properties of SAMs of $\pi$-conjugated molecules have not been similarly investigated. Thus, we

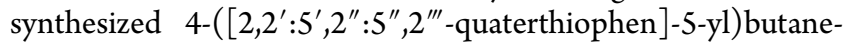
1-thiol (T4C4), a molecule containing both a flexible butanethiol chain to facilitate the formation of a densely packed monolayer and a rigid quarterthiophene to impart mechanical stability through relatively strong $\pi-\pi$ interactions. The molecular structure and the geometry of a CP-AFM junction are shown in Figure 1. We studied the mechanical and

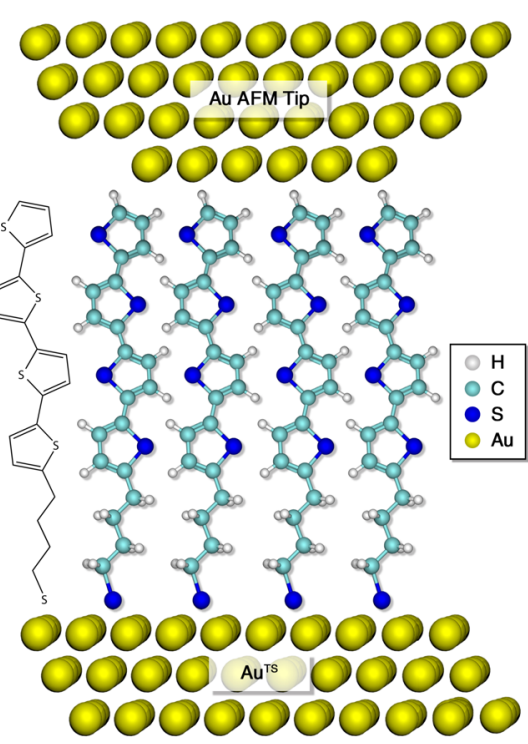

Figure 1. Representative schematic of molecular junction comprising T4C4 with a Au coated CP-AFM tip as top electrode and templatestripped $\mathrm{Au}$ as bottom electrode.

electrical properties of SAMs of T4C4 using CP-AFM and density functional theory (DFT). They are quantitatively more robust than SAMs of alkanethiolates, but the electrostatics of the junction respond to small distortions of the $\pi$-system. This robustness translates into junctions that are capable of withstanding larger bias windows than alkanethiolates in large-area junctions using eutectic Ga-In (EGaIn) topcontacts. $^{22}$

\section{METHODS}

Self-Assembled Monolayers. The formation of SAMs follows the reported methods. ${ }^{23} \mathrm{The} \mathrm{Ag}$ and Au substrates were prepared by template stripping (TS) described in details elsewhere. $^{24} 200 \mathrm{~nm}$ of $\mathrm{Ag}$ (99.99\%) and $100 \mathrm{~nm}$ of $\mathrm{Au}$ (99.99\%) were deposited by thermal deposition at $10^{-7} \mathrm{mbar}$ onto a 3" Silicon wafer (without adhesion layer). Glass substrates $(1 \mathrm{~cm} \times 1 \mathrm{~cm})$ were glued onto deposited metal by using UV-curable optical adhesive (Norland 61) with $300 \mathrm{~s}$ exposure of UV. All samples were made by incubation of freshly cleaved silver and gold substrates into either $3 \mathrm{mM}$ solution of the corresponding $n$-alkanethiols $(n=10,12,14,16)$ in ethanol or $0.5 \mathrm{mM}$ solution of $\mathrm{T} 4 \mathrm{C} 4$ in toluene at room temperature and kept inside a nitrogen flow box (in which the $\mathrm{O}_{2}$ was below $3 \%$ and the humidity was below $10 \%$ ) for $3 \mathrm{~h}$. Then the substrates of alkanethiols and T4C4 were rinsed by ethanol and toluene respectively and dried gently by $\mathrm{N}_{2}$. Prior to forming the SAMs, the solution was degassed by bubbling $\mathrm{N}_{2}$ for at least $20 \mathrm{~min}$ and all solution were kept under $\mathrm{N}_{2}$ atmosphere to prevent oxidation of thiol and $\mathrm{Ag}$ substrates. Characterization of the SAMs by water contact angles, ellipsometry, and ultraviolet photoelectron spectroscopy (UPS) is described in Supporting Information.

Current-Voltage Measurements. CP-AFM. I-V measurements were performed on a Bruker AFM Multimode MMAFM-2 equipped with a Peak Force TUNA Application Module (Bruker). The SAMs were contacted with an Aucoated silicon nitride tip with a nominal radius of $30 \mathrm{~nm}$ (NPG10 , Bruker; tip A, resonant frequency $=65 \mathrm{kHz}$, spring constant $=0.35 \mathrm{~N} / \mathrm{m}$; tip $\mathrm{B}$, resonant frequency $=23 \mathrm{kHz}$, spring constant $=0.12 \mathrm{~N} / \mathrm{m}$; tip $\mathrm{D}$, resonant frequency $=18 \mathrm{kHz}$, spring constant $=0.06 \mathrm{~N} / \mathrm{m}$; tip A was chosen in this work) in TUNA mode. The AFM tip was grounded and for all loading forces, $\mathrm{T} 4 \mathrm{C} 4$ on $\mathrm{Au}^{\mathrm{TS}}$ were biased from -1.0 to $+1.0 \mathrm{~V}$ and from +1.0 to $-1.0 \mathrm{~V}$ while $\mathrm{C} 10$ on were biased from -1.5 to $+1.5 \mathrm{~V}$ and from +1.5 to $-1.5 \mathrm{~V}$ on $\mathrm{Au}^{\mathrm{TS}}$ to record the $I-V$ curves: a max of 10 trace/retrace cycles per junction were performed and the top electrode was removed from SAMs between junctions. Between different samples, a new tip was used. The total number of $I-V$ traces recorded by CP-AFM is summarized in the Supporting Information, Table S3. It is difficult to determine $V_{\text {trans }}$ for an individual $I-V$ trace due to the inherent noise in the raw data. The peaks of Gaussian fits of histograms of $I$ for each value of $V$ at different loading forces obtained by CP-AFM were plotted and transformed into axes of $\ln \left(I / V^{2}\right)$ versus $1 / V$. The position of the $V_{\text {trans }}$ was determined manually by the center of the dips in the plots.

EGaln. The electrical measurement with the EGaIn, as well as sample preparation and handling, was performed in the nitrogen flow box in which $\mathrm{O}_{2}$ was maintained below 3\% and the humidity was kept below $10 \%$. At least two samples were examined for each molecule. The potential windows include the following: (1) $0 \mathrm{~V} \rightarrow 1 \mathrm{~V} \rightarrow-1 \mathrm{~V} \rightarrow 0 \mathrm{~V}$, steps of $0.05 \mathrm{~V}$; (2) $0 \mathrm{~V} \rightarrow 1.5 \mathrm{~V} \rightarrow-1.5 \mathrm{~V} \rightarrow 0 \mathrm{~V}$, steps of $0.1 \mathrm{~V}$; (3) $0 \mathrm{~V} \rightarrow 2 \mathrm{~V}$ $\rightarrow-2 \mathrm{~V} \rightarrow 0 \mathrm{~V}$, steps of $0.1 \mathrm{~V}$; (4) $0 \mathrm{~V} \rightarrow 2.5 \mathrm{~V} \rightarrow-2.5 \mathrm{~V} \rightarrow$ $0 \mathrm{~V}$, steps of $0.25 \mathrm{~V}$. A total of 20 trace/retrace cycles were measured for each junction, and shorts occuring during the cycles are counted for the failure of junction. The statistics of the stability test on EGaIn junctions are summarized in the Supporting Information, Table S5.

PeakForce QNM. Measurements of Young's modulus were performed on a Bruker Multimode MMAFM-2 in PeakForce QNM mode. The tip used in the measurement was ScanAsystAir from Bruker (resonant frequency, $70 \mathrm{kHz}$; spring constant, $0.4 \mathrm{~N} / \mathrm{m}$; tip radius, $2 \mathrm{~nm}$ ). Deflection sensitivity was calibrated by measuring 5 force curves on fused silica sample provided by Bruker and taking the average of the results. Spring constant was calibrated by thermal tune before and after the measurements. Tip radius was calibrated before and after the measurements using scanning electron microscope (SEM). Deformation under each force load was measured from five spots of the sample and averaged. Young's modulus was calculated by the DMT model from the averaged deformation of each force load.

DFT Calculations. All calculations were performed using Gaussian 09 software. Structures were first minimized by 
A

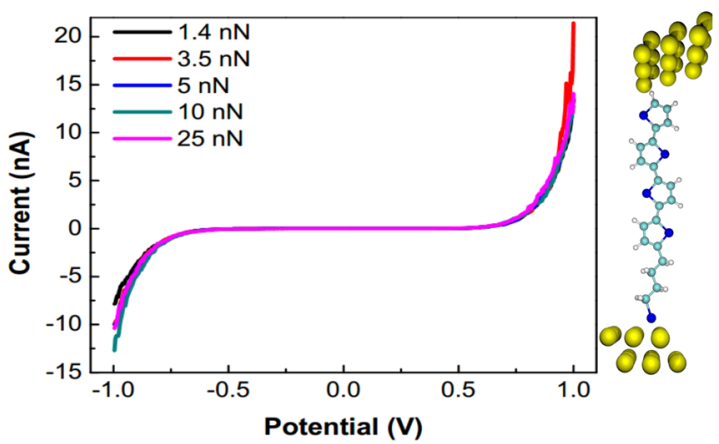

$\mathrm{B}$

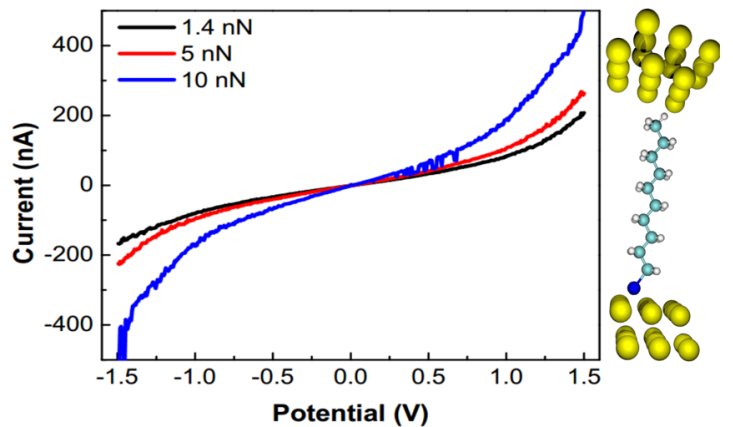

Figure 2. (A) $I-V$ plots of T4C4 with different forces: black for $1.4 \mathrm{nN}$, red for $3.5 \mathrm{nN}$, blue for $5 \mathrm{nN}$, dark cyan for $10 \mathrm{nN}$, and pink for $25 \mathrm{nN}$. (B) $I-V$ plots of $\mathrm{C} 10$ with different forces: black for $1.4 \mathrm{nN}$, red for $5 \mathrm{nN}$, and blue for $10 \mathrm{nN}$. Both SAMs were measured on Au ${ }^{\mathrm{TS}}$ substrates by CPAFM.

B3LYP/6-311G*; then, the single-point energies were computed by B3LYP/LANL2DZ. Single-molecule junctions were constructed by attaching the minimized structures to 17 or 18 -atom $\mathrm{Au}(111)$ clusters via the terminal sulfur atoms at hexagonal close-pack hollow sites at a distance of $1.75 \AA$ from the center of the hollow site. To calculate the energy difference $E_{\mathrm{f}}-E_{\text {HOPS }}$ and $E_{\mathrm{f}}-E_{\text {HOSS }}$, the value of $E_{\mathrm{f}}$ for Au electrodes was taken to be $-4.2 \mathrm{eV}$ from the UPS measurements.

\section{RESULTS AND DISCUSSION}

We synthesized T4C4, a " $\sigma-\pi$ " hybrid molecular structure containing both alkyl $(\sigma)$ and thiophene $(\pi)$ moieties according to the protocol described in the Supporting Information. We chose T4C4 because it is known to form densely packed SAMs. ${ }^{25-27}$ For comparison to previously reported mechanical studies, we used decanethiol (C10). We chose C10 specifically because the properties of SAMs of $\mathrm{C} 10$ have been studied extensively by CP-AFM. ${ }^{16,28,29}$ We prepared both templatestripped gold $\left(\mathrm{Au}^{\mathrm{TS}}\right)$ and silver $\left(\mathrm{Ag}^{\mathrm{TS}}\right)$ ultrasmooth electrodes following literature procedures template-stripping. ${ }^{24}$ These substrates are particularly well suited to large-area junctions ${ }^{30}$ and are compatible with CP-AFM. ${ }^{12}$

CP-AFM Measurements. We formed metal-moleculemetal junctions by placing the gold coated CP-AFM tip (denoted $\mathrm{Au}^{\mathrm{AFM}}$ ) with spring constant of $0.35 \mathrm{~N} \mathrm{~m}^{-1}$ and radius of $30 \mathrm{~nm}$ in a stationary point contact with the SAM under a controlled tip loading force, which translates into an applied pressure that depends on the radius of the tip; CP-AFM tips are larger than ordinary $\mathrm{Si}_{3} \mathrm{~N}_{4}$ tips due to the additional metallic layers. We refer to the molecular junctions as $\mathrm{Au}^{\mathrm{TS}} /$ $\mathrm{SAM} / / \mathrm{Au}^{\mathrm{AFM}}$, where "/" and "//" denote a covalent interface and a van der Waals contact, respectively. We measured the $I-$ $V$ characteristics of $\mathrm{Au}^{\mathrm{TS}} / \mathrm{C} 10 / / \mathrm{Au}^{\mathrm{AFM}}$ and $\mathrm{Au}^{\mathrm{TS}} / \mathrm{T} 4 \mathrm{C} 4 / /$ $\mathrm{Au}^{\mathrm{AFM}}$ junctions at low applied forces, which we define as 25 $\mathrm{nN}$ or less. Characteristic data are shown in Figure 2 for $\mathrm{C} 10$ and T4C4. (The $I-V$ curves for $\mathrm{C} 10$ at and above $25 \mathrm{nN}$ shorted when bias was applied and are, therefore, omitted from the figure.) The $I-V$ characteristics of C10 were sufficiently similar to published data to validate our measurement technique. ${ }^{16-20}$ The $I-V$ curves of T4C4 did not change at low forces (Figure 2A), passing approximately $10 \mathrm{nA}$ at $1 \mathrm{~V}$. The $I-V$ curves of $\mathrm{C} 10$, however, varied by about a factor of 2 , passing approximately $200 \mathrm{nA}$ at $1 \mathrm{~V}$ with a force of $10 \mathrm{nN}$ and $100 \mathrm{nA}$ at $1.4 \mathrm{nN}$. We were only able to measure T4C4 to $\pm 1 \mathrm{~V}$ without saturating the current amplifier, while we were able to measure $\mathrm{C} 10$ to $\pm 1.5 \mathrm{~V}$ using the low-gain amplifier because the absolute current in the intermediate-bias regime (i.e., where the $I-V$ dependence becomes exponential) increases more slowly for $\mathrm{C} 10$ than for T4C4. To verify the reproducibility of the data in Figure 2, we performed a statistical analysis by constructing histograms of $I$ at $\pm 1 \mathrm{~V}$ (Figures S6 and S7) and fitting them to Gaussian distributions. The data points with error bars reported throughout the main text and the Supporting Information are the peak and standard deviations, respectively, derived from such histograms.

The $I-V$ curves of T4C4 are sigmoidal, passing nearly invariant, low current in the linear, low-bias regime (below 0.5 $\mathrm{V}$ ) and increasing dramatically in the exponential, intermediatebias regime, which is consistent for $\pi$-conjugated (or $\sigma-\pi$ ) "molecular wire" molecules. ${ }^{31}$ The $I-V$ curves of $\mathrm{C} 10$ are sigmoidal, but increase throughout the low-bias regime, which is consistent for alkanethiols. 16,32 The evolution of the $I-V$ curves with increasing loading force can be caused by any combination of three factors: (1) the molecular tilt increases; (2) molecules in the SAM are deformed; and (3) the contact area increases. As mentioned above, the response of $\mathrm{C} 10$ is attributed mainly to the tilt angle, but $\mathrm{T} 4 \mathrm{C} 4$ showed no change at forces up to $30 \mathrm{nN}$ as can be seen in Figure 3. (Note that the dependence of pressure on loading force is nonlinear due to the dependence of contact-area on force, thus, the values across the top $X$-axis are only meant to show the range of pressures experienced by the SAM; see Supporting Information for

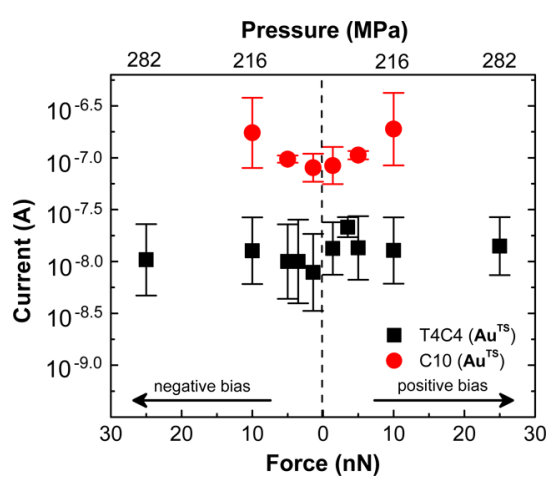

Figure 3. Current at $1.0 \mathrm{~V}$ versus loading force on $\mathrm{Au}^{\mathrm{TS}}$ plots: dark squares for $\mathrm{T} 4 \mathrm{C} 4$, red circles for $\mathrm{C} 10$. Each data point is the peak of Gaussian fit to a histogram of $I$ at that value of $V$. The error bars are standard deviations. The values listed on the top $X$-axis are the pressures calculated explicitly for the corresponding values of force on the bottom $X$-axis. 
A

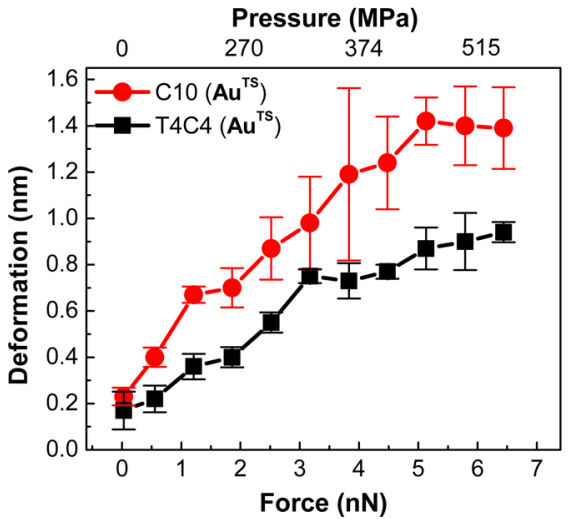

B

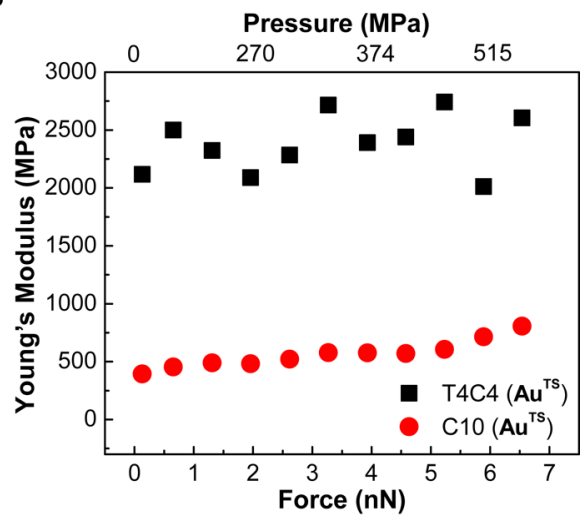

Figure 4. (A) Deformation of SAMs on $\mathrm{Au}^{\mathrm{TS}}$ : black squares for T4C4; red circles of C10. The error bars are standard deviations. (B) Young's modulus of SAMs: black squares for T4C4; red circles of C10. The values listed on the top $X$-axis are the pressures calculated explicitly for the corresponding values of force on the bottom $X$-axis.

A

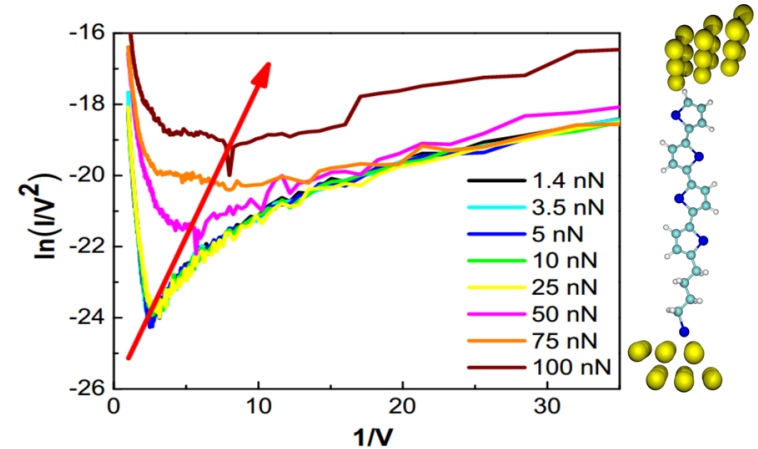

$\mathrm{B}$

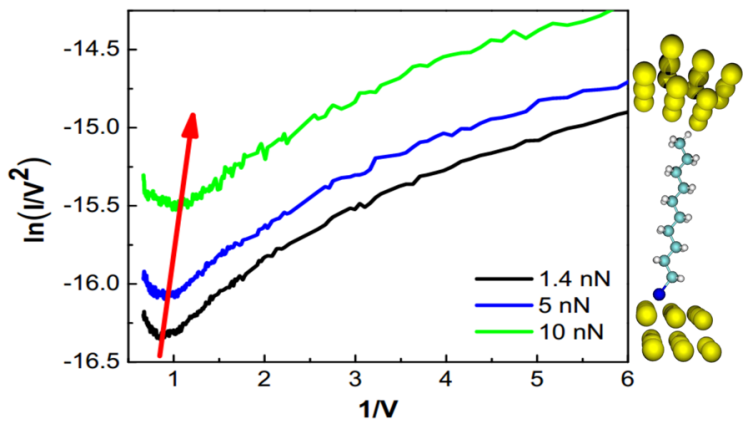

Figure 5. Transition voltage spectroscopy of $\mathrm{Au}^{\mathrm{TS}} / \mathrm{SAM} / / \mathrm{Au}$ junctions versus loading force from the peaks of Gaussian fits obtained by $\mathrm{CP}-\mathrm{AFM}$ at each loading force. (A) T4C4, 200 traces at each loading force. (B) C10, 30 traces at each loading force. The equivalent pressures for each force are shown in Table 1.

details.) This semilog scale plot compresses the data somewhat, but there is still a clear, increasing trend for $\mathrm{C} 10$ that is absent for T4C4 even up to $30 \mathrm{nN}$ (i.e., three times the loading force). At high force $(30-150 \mathrm{nN})$ the conductivity of T4C4 begins to increase, but $\mathrm{C} 10$ either shorts or saturates the current amplifier (both manifest as hitting the compliance limit) above $10 \mathrm{nN}$ (Figure S5). Thus, we measured SAMs of dodecanethiol (C12) in $\mathrm{Ag}^{\mathrm{TS}} / \mathrm{C} 12 / / \mathrm{Au}^{\mathrm{AFM}}$ and $\mathrm{Ag}^{\mathrm{TS}} / \mathrm{C} 12 / / \mathrm{EGaIn}$ junctions in order to compare the effects at high forces. We switched to $\mathrm{Ag}^{\mathrm{TS}}$ substrates for $\mathrm{C} 12$ to facilitate comparisons to literature reports for EGaIn, for which $\mathrm{Ag}^{\mathrm{TS}}$ is the preferred substrate as described below. ${ }^{23,33,34}$ We also measured $\mathrm{Ag}^{\mathrm{TS}} / \mathrm{T} 4 \mathrm{C} 4 / / \mathrm{Au}^{\mathrm{AFM}}$ and $\mathrm{Ag}^{\mathrm{TS}} / \mathrm{T} 4 \mathrm{C} 4 / / \mathrm{EGaIn}$ junctions for comparison. The increase in current of $\mathrm{Ag}^{\mathrm{TS}} / \mathrm{C} 12 / / \mathrm{Au}^{\mathrm{AFM}}$ as a function of loading force is even more dramatic than $\mathrm{Au}^{\mathrm{TS}} / \mathrm{C} 10 / / \mathrm{Au}^{\mathrm{AFM}}$ and $\mathrm{Ag}^{\mathrm{TS}} / \mathrm{C} 10 / / \mathrm{Au}^{\mathrm{AFM}}$, spanning 3 orders of magnitude up to $150 \mathrm{nN}$; see the Supporting Information, Figures S8 and S9, for details.

Mechanical Properties. In addition to $I-V$ measurements via $\mathrm{CP}$-AFM, we measured the mechanical properties of SAMs of T4C4 and $\mathrm{C} 10$ on $\mathrm{Au}^{\mathrm{TS}}$ using PeakForce QNM AFM. Figure 4A shows the deformation as a function of loading force up to 7 $\mathrm{nN}$. To enable a comparison between these data and CP-AFM data, we estimated the pressure applied to the SAM by considering the loading force and the radius of the tip (see the Supporting Information, Table S4). At loading forces below 3 $\mathrm{nN}$, the magnitudes and slopes are similar, but above $3 \mathrm{nN}$ the displacement of T4C4 begins to level off at approximately 0.8 $\mathrm{nm}$ while $\mathrm{C} 10$ continues to increase. We hypothesize that the inflection point in the T4C4 curve is caused by compression/ deformation of the butyl tail, which deforms at lower loading force than the quarterthiophene unit (but similar to $\mathrm{C} 10$ ). Figure 4B shows the Derjaguin-Muller-Toporov (DMT) Young's modulus (stiffness in the elastic region) over the same range of loading force. ${ }^{35}$ (There are no error bars because the Young's modulus was calculated from the average deformation of each force load using the DMT model.) The difference is unambiguous; the modulus of T4C4 is five times higher than $\mathrm{C} 10$, indicating that SAMs of T4C4 are considerably stiffer than SAMs of C10. Our measured values for C10 are also in good agreement with the moduli for SAMs of alkanethiolates reported previously; $280 \mathrm{MPa}$ for octanethiol (C8) and 860 $\mathrm{MPa}$ for $\mathrm{C} 12 .{ }^{36}$ From the electrical and mechanical measurements, we conclude that SAMs of T4C4 are more mechanically robust than $\mathrm{C} 10$, which translates into more stable conductance across a wider range of loading force; however, conductance alone does not provide much insight into the electrostatics of the junctions or address the question of why the $I-V$ characteristics of $\mathrm{T} 4 \mathrm{C} 4$ are stable despite deforming considerably at low loading forces.

Transition Voltage Spectroscopy. Transition voltage spectroscopy (TVS) is a useful tool to gain insights into the electrostatics of molecular junctions by providing an indirect measure of $\phi$, the offset between $E_{\mathrm{f}}$ and the frontier orbital that 
participates most strongly in tunneling transport (the highestoccupied state for both $\mathrm{C} 10$ and T4C4, i.e., hole transport). The transition voltage $V_{\text {trans }}$ corresponds to the transition from ohmic, low-bias conduction to exponential/hyperlinear conduction at intermediate bias, which can be estimated by replotting the $I-V$ curves and looking for minimums. The value of $V_{\text {trans }}$ is proportional to the height of the tunneling barrier imposed by $\phi$. Shifts in $V_{\text {trans }}$, therefore, reveal changes to $\phi$, which is a function of the electrostatics (i.e., level-alignment) near $E_{\mathrm{f}}$. These shifts can occur independently of changes in conductance, either because they are below the threshold for detection or are offset by other changes, for example, the barrier width, which is related to the distance between the electrodes and, therefore, decreases as the SAM deforms. To compute $V_{\text {trans }}$, we plotted $\ln \left(I V^{-2}\right)$ vs $V^{-1}$ using the peaks of Gaussian fits of histograms of $I$ for each value of $V$ at different loading forces (200 traces for T4C4 and 30 for C10) and recorded the center of the dips in the plots. These data are plotted in Figure 5 and summarized in Table 1 . At loading forces above $75 \mathrm{nN}$ the dips were not very pronounced, but they were well-resolved at all other forces, revealing clear differences between T4C4 and C10.

Table 1. Measured Values of $V_{\text {trans }}^{+}$at Different Loading Forces

\begin{tabular}{cccc} 
& & \multicolumn{2}{c}{$V_{\text {trans }}^{+}(\mathrm{V})$} \\
\cline { 3 - 4 } pressure $(\mathrm{MPa})$ & force $(\mathrm{nN})$ & $\mathrm{T} 4 \mathrm{C} 4$ & $\mathrm{C} 10$ \\
163.12 & 1.4 & 0.4 & 1.20 \\
177.35 & 3.5 & 0.4 & - \\
186.94 & 5.0 & 0.4 & 1.10 \\
215.93 & 10 & 0.38 & 0.95 \\
282.28 & 25 & 0.34 & - \\
352.43 & 50 & 0.18 & - \\
396.49 & 75 & 0.14 & - \\
426.72 & 100 & 0.13 & - \\
\hline
\end{tabular}

The trend for C10 shown in Figure 5B and Table 1 is in excellent agreement with literature values; $V_{\text {trans }}^{+}\left(V_{\text {trans }}\right.$ at positive bias) decreases from a maximum of 1.20 to $0.95 \mathrm{~V}$, a change of approximately $20 \%$. Table 2 compares literature

Table 2. Comparison of $V_{\text {trans }}^{+}$of Alkanethiols on $\mathrm{Au}$ Substrate at Low Loading Forces to Literature Values

\begin{tabular}{llcl} 
& \multicolumn{3}{c}{$V_{\text {trans }}^{+}(\mathrm{V})$} \\
\cline { 2 - 4 } & $\mathrm{C} 8$ & $\mathrm{C} 10$ & $\mathrm{C} 12$ \\
this work & - & 1.20 & - \\
ref 29 & 1.28 & 1.27 & 1.20 \\
ref 37 & 1.21 & - & 1.33 \\
\hline
\end{tabular}

values of $V_{\text {trans }}^{+}$for $\mathrm{C} 10, \mathrm{C} 12$ and $\mathrm{C} 8$ at low loading force to our value for C10; these values, which are typically 1.10 to $1.40 \mathrm{~V}$ for alkanethiols, are also in excellent agreement. ${ }^{29,37}$ Because $V_{\text {trans }}$ is proportional to $\phi$ and $E_{\mathrm{f}}$ is invariant (i.e., the value for $\mathrm{Au}^{\mathrm{TS}}$,) $V_{\text {trans }}$ is almost always smaller for $\pi$-conjugated molecules than for alkanethiols by virtue of the fact that the HOMO tends to lie closer to $E_{\mathrm{f}}{ }^{38,39}$ Indeed, $V_{\text {trans }}^{+}$for T4C4 is about one-third the value of $\mathrm{C} 10$. Moreover, it decreases from 0.4 to $0.13 \mathrm{~V}$, a change of approximately $70 \%$ over a range of $1.4 \mathrm{nN}$ to $100 \mathrm{nN}$. From $1.4 \mathrm{nN}$ to $10 \mathrm{nN}$, the range over which $\mathrm{C} 10$ could be measured, T4C4 only changes by approximately $5 \%$, compared to $20 \%$ for C10. Thus, the changes in conductance in both SAMs correspond to a lowering of the barrier height, but it requires about 1 order of magnitude more loading force to induce a change in $\mathrm{T} 4 \mathrm{C} 4$ as compared to C10. Given the substantial differences in chemical structure and mechanical properties, it is unlikely that the cause of the shifts in $V_{\text {trans }}$ are the same for $\mathrm{T} 4 \mathrm{C} 4$ as they are for $\mathrm{C} 10$ (i.e., increased tilt angle).

DFT Calculations. For insights into the electrostatics of SAMs of T4C4 under deformation, we constructed model junctions and computed their properties using DFT. The model junctions consist of single molecules spanning two clusters of $\mathrm{Au}$ atoms; these are not meant as direct simulations of $\mathrm{Au}^{\mathrm{TS}} / \mathrm{SAM} / / \mathrm{Au}$ junctions, rather, they are computationally accessible models from which we can compute trends to compare to experimental data. First, we optimized the geometry of the molecule in the gas-phase using B3LYP/6$311 \mathrm{G}^{*}$. Given the coplanar geometry of the quarterthiophene moiety and the tendency for alkanes to adopt a trans-extended conformation in SAMs, this geometry is a reasonable approximation for T4C4 in a SAM. Second, we attached a cluster of $\mathrm{Au}$ at a hollow site via the thiol anchor on one end and positioned an identical cluster above the terminal thiophene ring/methyl group at the other end. (The Au-S and $\mathrm{Au}$-thiophene distances do have a small effect on the computed electrostatics, but they are kept constant across all calculations such that the effect is constant.)

Finally, we computed point energies using B3LYP/ LANL2DZ for the molecule before and after attaching the metal electrodes to compare the orbital energies and isoplots of the molecule in gas phase and in the model junctions, respectively. To model the deformation of the SAM, we distorted the T4C4 molecules in the model junctions systematically either by hand or by using displacements predicted from frequency calculations. The figure of merit of these calculations is the offset between the metal Fermi level and the highest-occupied $\pi$-state (HOPS) of T4C4 $\left(E_{\mathrm{f}}-\right.$ $\left.E_{\mathrm{HOPS}}\right)$, which is a direct approximation of $\phi$ and, therefore, will vary accordingly with $V_{\text {trans }}$. Because these are Gaussian (i.e., discrete, aperiodic) calculations the "HOMO" corresponds to $E_{\mathrm{f}}$, thus we locate the HOPS by comparing the model junction to the gas-phase calculation. Figure S11 shows isoplots of the HOPS for T4C4 and the highest-occupied $\sigma$-state (HOSS) for C10 obtained using this method.

We estimated $E_{\mathrm{f}}-E_{\mathrm{HOPS}}$ of SAMs of T4C4 on $\mathrm{Au}^{\mathrm{TS}}$ and $\mathrm{Ag}^{\mathrm{TS}}$ experimentally from ultraviolet photoelectron spectroscopy (UPS) data according to ref 39 (Table 3). To relate the

Table 3. Energy Levels Determined by UPS

\begin{tabular}{cccccc} 
& \multicolumn{2}{c}{ HOPS $(\mathrm{eV})$} & & \multicolumn{2}{c}{$E_{\mathrm{f}}-E_{\text {HOPS }}(\mathrm{eV})$} \\
\cline { 2 - 3 } \cline { 5 - 6 } $\mathrm{N} 4 \mathrm{C} 4$ on $\mathrm{Au}$ & -5.42 & -4.88 & & 1.23 & 0.68 \\
$\mathrm{~T} 4 \mathrm{C} 4$ on $\mathrm{Ag}$ & -5.25 & -4.71 & & 1.31 & 0.77 \\
\hline
\end{tabular}

DFT calculations to experimental data, we computed $E_{\mathrm{f}}-$ $E_{\text {HOPS }}$ using the value of $E_{\mathrm{f}}$ from UPS and the value of $E_{\mathrm{HOPS}}$ from DFT of the minimized geometry of T4C4 in a model Au/ $\mathrm{T} 4 \mathrm{C} 4 / \mathrm{Au}$ junction. This method produced excellent agreement for $E_{\mathrm{f}}-E_{\mathrm{HOPS}}$ between UPS and DFT.

Figure 6 shows $E_{\mathrm{f}}-E_{\mathrm{HOPS}}$ of model junction as a function of in-plane bending. Unsurprisingly, there is hardly any effect on C10; however, the response of $\mathrm{T} 4 \mathrm{C} 4$ is nonlinear, increasing at first and then rapidly decreasing. The initial increase is due to 
A

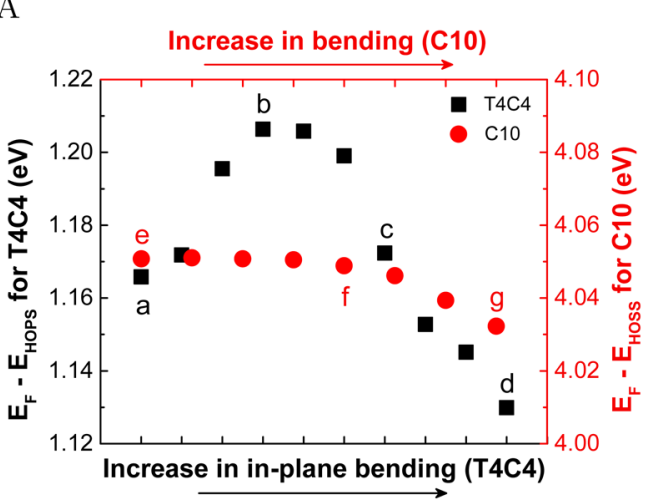

$\mathrm{B}$

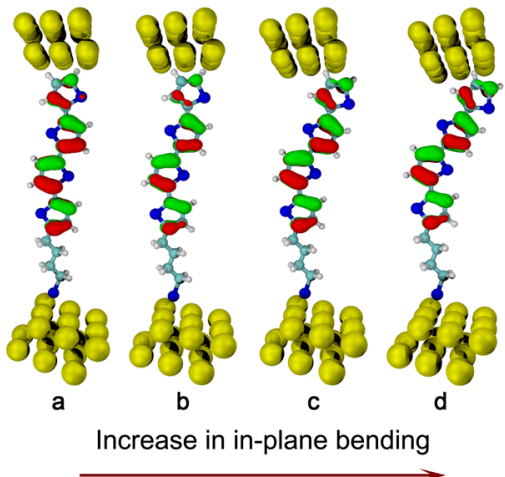

Figure 6. (A) Shift in the energy of $E_{\mathrm{f}}-E_{\mathrm{HOPS}}$ of $\mathrm{Au}^{\mathrm{TS}} / \mathrm{SAM} / / \mathrm{Au}$ model junctions with the increased in-plane bending of the T4C4 molecules (black squares), and $E_{\mathrm{f}}-E_{\mathrm{HOSS}}$ with the increased bending of $\mathrm{C} 10$ alkanethiol molecules (red dots), relative to their equilibrium geometries. The labeled data points (a, b, c, and d) correspond to the energies of the T4C4 geometries showed in the bottom schematic (B). The first points a and e correspond to optimized geometries of $\mathrm{T} 4 \mathrm{C} 4$ and $\mathrm{C} 10$ molecules, respectively. The geometries corresponding to the data points e, $\mathrm{f}$, and $\mathrm{g}$ and further details are given in the Supporting Information. The $E_{\mathrm{f}}$ of $\mathrm{Au}$ electrodes was set to $-4.20 \mathrm{eV}$ for these plots from the UPS measurements.

A

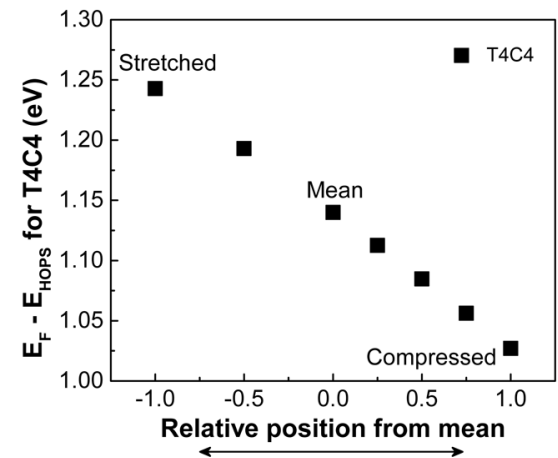

B

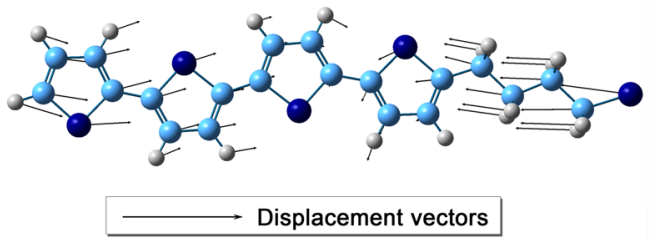

Figure 7. (A) Shift in the energy of $E_{\mathrm{f}}-E_{\mathrm{HOPS}}$ of $\mathrm{Au}^{\mathrm{TS}} / \mathrm{T} 4 \mathrm{C} 4 / / \mathrm{Au}$ model junctions as a function of the displacement of atoms along vibrational vectors from frequency calculations. The points on $X$-axis span from -1 (fully stretched geometry) to +1 (fully compressed geometry), where 0 corresponds to the equilibrium geometry. (B) Arrows represent the displacement vectors of individual atoms as they vibrate. The $E_{\mathrm{f}}$ of Au electrodes was set to $-4.20 \mathrm{eV}$ for these plots from the UPS measurements.

the decrease in orbital overlap in the $\pi$-system, which lowers the energy of the HOPS (the total energy still increases). It is not clear why $E_{\mathrm{f}}-E_{\mathrm{HOPS}}$ then decreases, but since we did not observe any increase in $V_{\text {trans }}$ experimentally, we conclude that in-plane bending (a relatively high-energy deformation, particularly in a SAM) does not play a large role in the mechanical deformation of SAMs of T4C4; we cannot exclude its contribution to $\mathrm{C} 10$, however. The exact bond angles of each conformation are shown in Figure S12 and Tables S6 and S7.

In-plane bending is a relatively high-energy process. Deformations in which atoms are allowed to displace along all vibrational vectors are generally lower-energy processes, but are more difficult to rationalize because it translates a compressive force (from the AFM tip) into motion in all directions within a SAM. Nonetheless, molecules of T4C4 stretched and compressed along these vectors show a linear response of $E_{\mathrm{f}}-E_{\mathrm{HOPS}}$ as a function of relative displacement as is shown in Figure 7. This response (as we go from "stretched" to "compressed" forms) also correctly predicts the direction of change in $V_{\text {trans }}$. Given the high Young's modulus and relatively small tip displacement, we hypothesize that the shifts in $V_{\text {trans }}$ for $\mathrm{Au}^{\mathrm{TS}} / \mathrm{T} 4 \mathrm{C} 4 / / \mathrm{Au}$ junctions are, therefore, the result of compressing molecules of $\mathrm{T} 4 \mathrm{C} 4$ along displacement vectors corresponding to vibrational modes that are allowed by the constraints of the SAM. This is a very different mechanism from that of $\mathrm{C} 10$ and provides a coherent explanation for the change in conductance that occurs at high loading forces. Other bending and twisting modes yielded either no change or an increase in $V_{\text {trans; }}$; see Figures S13 and S14 and the Supporting Information for further details.

Stability of Large-Area Junctions. The studies enumerated above probe areas on the order of tens of $\mathrm{nm}^{2}$ to give insight into the bulk mechanical properties of a SAM (e.g., stiffness,) however, the electrical properties that SAMs exhibit in large-area junctions include the influence of defects (e.g., local disorder) driven by inhomogeneities in the substrate, chemical impurities and grain boundaries. ${ }^{24,40-42}$ Shorter alkanethiols exhibit more resilience to defects because they are more liquid-like; ${ }^{43}$ however, when a voltage is applied to a large-area junction, a substantial electrostatic pressure develops that can deform and induce the reorganization ${ }^{44}$ in which case the stiffness of longer alkyl chains is advantageous. We hypothesize that there is, therefore, a relationship between the mechanical stability of a SAM and its breakdown voltage; SAMs that can withstand higher electrostatic pressures should form large-area tunneling junctions that resist shorting at high bias. There is no consensus on the mechanism of failure of 


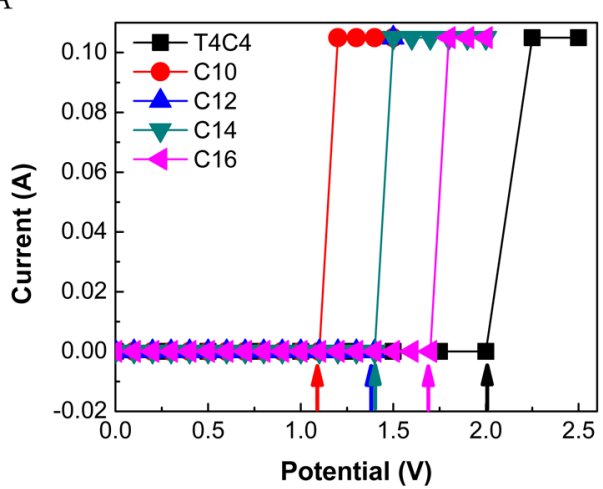

B

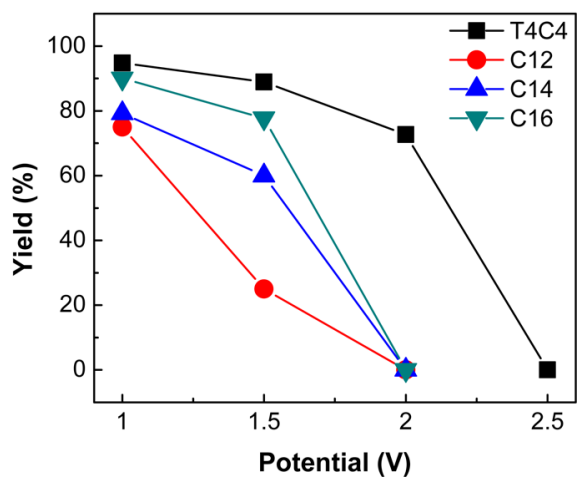

Figure 8. (A) Representative $I-V$ plots show the breakdown voltage of $\mathrm{Ag}^{\mathrm{TS}} / \mathrm{SAM} / / \mathrm{EGaIn}$ junctions comprising $\mathrm{T} 4 \mathrm{C} 4, \mathrm{C} 10, \mathrm{C} 12, \mathrm{C} 14$, and $\mathrm{C} 16$. (B) Yield of nonshorting junctions as a function of potential window.

large-area junctions at high bias, which could be (1) entirely a function of the ability of a SAM to resist penetration by the topcontact, (2) the migration of metal atoms from the bottomcontact, e.g., the formation of filaments of $\mathrm{Au}$, (3) electrochemical instability, or (4) some combination of the three. A clear correlation between breakdown voltage and the mechanical robustness of T4C4 would imply that mechanism 1 is dominant because the electrochemical window of T4C4 is much smaller than that of an alkanethiol. Extending the potential window in which a SAM can operate in a large-area junction is particularly relevant to molecule diodes ${ }^{45}$ such as SAMs incorporating ferrocenyl, ${ }^{46,47}$ bypyridyl, ${ }^{48,49}$ pyrimydyl, ${ }^{14}$ and fullerene ${ }^{50}$ moieties because the degree of rectification tends to scale with bias and they function under bias in integrated circuits. ${ }^{10}$

To investigate the influence of mechanical stability on breakdown voltages in large-area molecular junctions, we formed $\mathrm{Ag}^{\mathrm{TS}} / \mathrm{SAM} / / \mathrm{EGaIn}$ junctions ${ }^{22}$ of T4C4, C12, tetradecanethiol (C14), and hexadecanethiol (C16). As mentioned above, we chose $\mathrm{Ag}^{\mathrm{TS}}$ because it is the most commonly reported substrate for EGaIn top-contacts. We swept junctions of each SAM through increasing bias windows and recorded the frequency of shorts, defined by the sudden increase in current to the compliance limit of the instrument. Figure $8 \mathrm{~A}$ shows representative $I-V$ plots (on a linear scale) revealing a clear trend of increasing breakdown potential: T4C4 $>\mathrm{C} 16>\mathrm{C} 14 \approx \mathrm{C} 12>\mathrm{C} 10$. Figure $8 \mathrm{~B}$ shows the percent-yield of nonshorting junctions scanned from $\pm 1, \pm 1.5, \pm 2$, and \pm 2.5 V (see Table S5 for details). All SAMs shorted $100 \%$ of the time at $\pm 2.5 \mathrm{~V}$, but only $20 \%$ of junctions comprising T4C4 shorted at $\pm 2 \mathrm{~V}$, whereas $100 \%$ of junctions comprising C12, $\mathrm{C} 14$ and $\mathrm{C} 16$ shorted. At \pm 1 and $\pm 1.5 \mathrm{~V}$ there is a clear trend of increasing percentage of shorts: $\mathrm{C} 12>\mathrm{C} 14>\mathrm{C} 16>\mathrm{T} 4 \mathrm{C} 4$. This trend supports the hypothesis that the primary mode of failure of these $\mathrm{Ag}^{\mathrm{TS}} / \mathrm{SAM} / / \mathrm{EGaIn}$ junctions is mechanical failure due to electrostatic pressure from the applied bias; the mechanical robustness of SAMs of alkanethiolates scales with chain-length, but T4C4 is considerably more robust than a SAM of alkanethiolates of any number of carbons up to at least C16.

\section{CONCLUSION}

Technological applications of molecular electronics in the medium-term will almost certainly utilize SAMs; they simplify fabrication and large-area junctions, in particular, can already be incorporated into integrated circuits and wafer-scale manufac- turing processes. The usefulness of molecular tunneling junctions derives from the nonlinear dependence of $I-V$ characteristics on the conformation and electronic structure of the molecules. However, the $I-V$ properties of bottom-up junctions comprising SAMs are affected by mechanical force. For SAMs of alkanethiolates, mechanical forces disturb the packing of the SAM, causing tilt angles to increase. We have shown that the electronic structure of $\pi$-conjugated molecules (i.e., the electrostatics of the junction) is also directly affected by mechanical force. Thus, it is important to develop an understanding of this relationship and relate it to molecular structure such that the mechanical properties of a SAM and how a tunneling junction responds to forces can be tailored synthetically both to increase the robustness and stability of junctions and to develop devices that respond to mechanical inputs.

We have shown that SAMs of a $\sigma-\pi$ molecule designed to maximize intermolecular interactions, $\mathrm{T} 4 \mathrm{C} 4$, are significantly more mechanically robust than SAMs of alkanethiolates. Selfassembled monolayers of T4C4 undergo less deformation as a function of loading force by AFM and Young's modulus is approximately five times higher. At relatively low loading forces, tunneling junctions comprising SAMs of T4C4 show no changes in conductance or values of $V_{\text {trans }}$; SAMs of C10 show significant changes. At higher loading forces than SAMs of C10 are capable of withstanding, junctions comprising T4C4 begin to show differences. Our DFT calculations suggest that these changes are due to force-induced distortions of the $\pi$-system and not, as is the case for C10, a change in tilt angle; the AFM tip does not penetrate SAMs of T4C4 as it does C10. The mechanical stability of T4C4 translates into a higher breakdown potential in large-area tunneling junctions with EGaIn topcontacts. This observation suggests that electrostatic pressure plays a significant role in the shorting of molecular tunneling junctions at high bias; SAMs of T4C4 are more mechanically robust than SAMs of alkanethiolates, but T4C4 is significantly less electrochemically stable than an alkanethiol. Our results demonstrate that it is possible to design molecules that maximize mechanical properties and breakdown voltages in large-area tunneling junctions comprising SAMs.

\section{ASSOCIATED CONTENT}

\section{Supporting Information}

The Supporting Information is available free of charge on the ACS Publications website at DOI: 10.1021/acs.jpcc.7b03853. 
Details of synthesis and characterization of molecules and SAMs, details on EGaIn and CP-AFM measurements, and details on DFT calculations and parameters used, including more deformation simulations (PDF)

\section{AUTHOR INFORMATION}

\section{Corresponding Author}

*(R.C.C.) E-mail: r.c.chiechi@rug.nl.

ORCID $\odot$

Andreas Herrmann: 0000-0002-8886-0894

Ryan C. Chiechi: 0000-0002-0895-2095

\section{Present Address}

${ }^{\S}$ Department of Chemistry, Northwestern University, 2145 Sheridan Road, Evanston, Illinois 60208-3113, United States

\section{Author Contributions}

${ }^{\perp}$ These authors contributed equally to this work.

\section{Notes}

The authors declare no competing financial interest.

\section{ACKNOWLEDGMENTS}

R.C.C. and Y.Z. acknowledge the European Research Council for the ERC Starting Grant 335473 (MOLECSYNCON). X.Q. acknowledges the Zernike Institute for Advanced Materials "Dieptestrategie." We thank Prof. J. C. Hummelen for providing a quarterthiophene synthon. We thank the Center for Information Technology of the University of Groningen for their support and for providing access to the Peregrine high performance computing cluster.

\section{REFERENCES}

(1) Service, R. F. The Brain Chip. Science 2014, 345, 614-616.

(2) Xiang, D.; Wang, X.; Jia, C.; Lee, T.; Guo, X. Molecular-Scale Electronics: From Concept to Function. Chem. Rev. 2016, 116, 43184440.

(3) Wang, G.; Kim, Y.; Na, S.-I.; Kahng, Y. H.; Ku, J.; Park, S.; Jang, Y. H.; Kim, D.-Y.; Lee, T. Investigation of the Transition Voltage Spectra of Molecular Junctions Considering Frontier Molecular Orbitals and the Asymmetric Coupling Effect. J. Phys. Chem. C 2011, 115, 17979-17985.

(4) Cui, X. D.; Primak, a.; Zarate, X.; Tomfohr, J.; Sankey, O. F.; Moore, a. L.; Moore, T. a.; Gust, D.; Harris, G.; Lindsay, S. M. Reproducible Measurement of Single-Molecule Conductivity. Science 2001, 294, 571-574.

(5) Tao, N. J. Electron Transport in Molecular Junctions. Nat. Nanotechnol. 2006, 1, 173-181.

(6) Akkerman, H. B.; Blom, P. W. M.; de Leeuw, D. M.; de Boer, B. Towards Molecular Electronics With Large-Area Molecular Junctions. Nature 2006, 441, 69-72.

(7) Wang, G.; Kim, Y.; Choe, M.; Kim, T.-W.; Lee, T. A New Approach for Molecular Electronic Junctions With a Multilayer Graphene Electrode. Adv. Mater. 2011, 23, 755-760.

(8) Zhang, Y.; Zhao, Z.; Fracasso, D.; Chiechi, R. C. Bottom-Up Molecular Tunneling Junctions Formed by Self-Assembly. Isr. J. Chem. 2014, 54, 513-533.

(9) van Hal, P. A.; Smits, E. C. P.; Geuns, T. C. T.; Akkerman, H. B.; De Brito, B. C.; Perissinotto, S.; Lanzani, G.; Kronemeijer, A. J.; Geskin, V.; Cornil, J.; et al. Upscaling, Integration and Electrical Characterization of Molecular Junctions. Nat. Nanotechnol. 2008, 3, 749-754.

(10) Wan, A.; Suchand Sangeeth, C. S.; Wang, L.; Yuan, L.; Jiang, L.; Nijhuis, C. A. Arrays of High Quality SAM-based Junctions and Their Application in Molecular Diode Based Logic. Nanoscale 2015, 7, 19547-19556.
(11) Kovalchuk, A.; Abu-Husein, T.; Fracasso, D.; Egger, D. A.; Zojer, E.; Zharnikov, M.; Terfort, A.; Chiechi, R. C. Transition Voltages Respond to Synthetic Reorientation of Embedded Dipoles in Self-Assembled Monolayers. Chem. Sci. 2016, 7, 781-787.

(12) Carlotti, M.; Kovalchuk, A.; Wächter, T.; Qiu, X.; Zharnikov, M.; Chiechi, R. C. Conformation-Driven Quantum Interference Effects Mediated by Through-Space Conjugation in Self-Assembled Monolayers. Nat. Commun. 2016, 7, 13904.

(13) Nerngchamnong, N.; Yuan, L.; Qi, D.-C.; Li, J.; Thompson, D.; Nijhuis, C. A. The Role of Van Der Waals Forces in the Performance of Molecular Diodes. Nat. Nanotechnol. 2013, 8, 113-118.

(14) Kovalchuk, A.; Egger, D. A.; Abu-Husein, T.; Zojer, E.; Terfort, A.; Chiechi, R. C. Dipole-Induced Asymmetric Conduction in Tunneling Junctions Comprising Self-Assembled Monolayers. RSC Adv. 2016, 6, 69479-69483.

(15) Venkataraman, L.; Klare, J. E.; Nuckolls, C.; Hybertsen, M. S.; Steigerwald, M. L. Dependence of Single-Molecule Junction Conductance on Molecular Conformation. Nature 2006, 442, 904907.

(16) Wold, D. J.; Frisbie, C. D. Fabrication and Characterization of Metal-Molecule-Metal Junctions by Conducting Probe Atomic Force Microscopy. J. Am. Chem. Soc. 2001, 123, 5549-5556.

(17) Song, H.; Lee, H.; Lee, T. Intermolecular Chain-To-Chain Tunneling in Metal-Alkanethiol-Metal Junctions. J. Am. Chem. Soc. 2007, 129, 3806-3807.

(18) Qi, Y.; Ratera, I.; Park, J. Y.; Ashby, P. D.; Quek, S. Y.; Neaton, J. B.; Salmeron, M. Mechanical and Charge Transport Properties of Alkanethiol Self-Assembled Monolayers on a $\mathrm{Au}(111)$ Surface: The Role of Molecular Tilt. Langmuir 2008, 24, 2219-2223.

(19) Wang, G.; Kim, T.-W.; Jo, G.; Lee, T. Enhancement of Field Emission Transport by Molecular Tilt Configuration in MetalMolecule-Metal Junctions. J. Am. Chem. Soc. 2009, 131, 5980-5985.

(20) Smaali, K.; Desbief, S.; Foti, G.; Frederiksen, T.; Sanchez-Portal, D.; Arnau, A.; Nys, J. P.; Leclere, P.; Vuillaume, D.; Clement, N. On the Mechanical and Electronic Properties of Thiolated Gold Nanocrystals. Nanoscale 2015, 7, 1809-1819.

(21) Beebe, J. M.; Kim, B.; Gadzuk, J. W.; Daniel Frisbie, C.; Kushmerick, J. G. Transition From Direct Tunneling to Field Emission in Metal-Molecule-Metal Junctions. Phys. Rev. Lett. 2006, 97, 026801.

(22) Chiechi, R. C.; Weiss, E. A.; Dickey, M. D.; Whitesides, G. M. Eutectic Gallium-Indium (EGaIn): A Moldable Liquid Metal for Electrical Characterization of Self-Assembled Monolayers. Angew. Chem. 2008, 120, 148-150.

(23) Jiang, L.; Sangeeth, C. S. S.; Nijhuis, C. A. The Origin of the Odd-Even Effect in the Tunneling Rates Across EGaIn Junctions With Self-Assembled Monolayers (SAMs) of N-Alkanethiolates. J. Am. Chem. Soc. 2015, 137, 10659-10667.

(24) Weiss, E. A.; Kaufman, G. K.; Kriebel, J. K.; Li, Z.; Schalek, R.; Whitesides, G. M. Si/SiO2-Templated Formation ofUltraflat Metal Surfaces on Glass, Polymer, and Solder Supports: Their Use as Substrates for Self-Assembled Monolayers. Langmuir 2007, 23, 96869694.

(25) De Boer, B.; Meng, H.; Perepichka, D. F.; Zheng, J.; Frank, M. M.; Chabal, Y. J.; Bao, Z. Synthesis and Characterization of Conjugated Mono- And Dithiol Oligomers and Characterization of Their Self-Assembled Monolayers. Langmuir 2003, 19, 4272-4284.

(26) Liedberg, B.; Yang, Z.; Engquist, I.; Wirde, M.; Gelius, U.; Götz, G.; Bäuerle, P.; Rummel, R.-M.; Ziegler, C.; Göpel, W. Self-Assembly of $\alpha$-Functionalized Terthiophenes on Gold. J. Phys. Chem. B 1997, $101,5951-5962$.

(27) Kato, H. S.; Murakami, Y.; Kiriyama, Y.; Saitoh, R.; Ueba, T.; Yamada, T.; Ie, Y.; Aso, Y.; Munakata, T. Decay of the Exciton in Quaterthiophene-Terminated Alkanethiolate Self-Assembled Monolayers on $\mathrm{Au}(111)$. J. Phys. Chem. C 2015, 119, 7400-7407.

(28) Beebe, J. M.; Engelkes, V. B.; Miller, L. L.; Frisbie, C. D. Contact Resistance in Metal-Molecule-Metal Junctions Based on Aliphatic SAMs: Effects of Surface Linker and Metal Work Function. J. Am. Chem. Soc. 2002, 124, 11268-11269. 
(29) Beebe, J. M.; Kim, B.; Frisbie, C. D.; Kushmerick, J. G. Measuring Relative Barrier Heights in Molecular Electronic Junctions With Transition Voltage Spectroscopy. ACS Nano 2008, 2, 827-832.

(30) Weiss, E. A.; Chiechi, R. C.; Kaufman, G. K.; Kriebel, J. K.; Li, Z.; Duati, M.; Rampi, M. A.; Whitesides, G. M. Influence of Defects on the Electrical Characteristics of Mercury-Drop Junctions: SelfAssembled Monolayers of N-Alkanethiolates on Rough and Smooth Silver. J. Am. Chem. Soc. 2007, 129, 4336-4349.

(31) Choi, S. H.; Risko, C.; Delgado, M. C. R.; Kim, B.; Brédas, J.-L.; Frisbie, C. D. Transition From Tunneling to Hopping Transport in Long, Conjugated Oligo-Imine Wires Connected to Metals. J. Am. Chem. Soc. 2010, 132, 4358-4368.

(32) Xiang, D.; Zhang, Y.; Pyatkov, F.; Offenhäusser, A.; Mayer, D. Gap Size Dependent Transition From Direct Tunneling to Field Emission in Single Molecule Junctions. Chem. Commun. 2011, 47, $4760-4762$.

(33) Simeone, F. C.; Yoon, H. J.; Thuo, M. M.; Barber, J. R.; Smith, B.; Whitesides, G. M. Defining the Value of Injection Current and Effective Electrical Contact Area for EGaIn-Based Molecular Tunneling Junctions. J. Am. Chem. Soc. 2013, 135, 18131-18144.

(34) Carlotti, M.; Degen, M.; Zhang, Y.; Chiechi, R. C. Pronounced Environmental Effects on Injection Currents in EGaIn Tunneling Junctions Comprising Self-Assembled Monolayers. J. Phys. Chem. C 2016, 120, 20437-20445.

(35) Dokukin, M. E.; Sokolov, I. Quantitative Mapping of the Elastic Modulus of Soft Materials with HarmoniX and PeakForce QNM AFM Modes. Langmuir 2012, 28, 16060-16071.

(36) DelRio, F. W.; Jaye, C.; Fischer, D. A.; Cook, R. F. Elastic and Adhesive Properties of Alkanethiol Self-Assembled Monolayers on Gold. Appl. Phys. Lett. 2009, 94, 131909.

(37) Ricœur, G.; Lenfant, S.; Guérin, D.; Vuillaume, D. Molecule/ Electrode Interface Energetics in Molecular Junction: A "Transition Voltage Spectroscopy" Study. J. Phys. Chem. C 2012, 116, 2072220730.

(38) Xie, Z.; Bâldea, I.; Smith, C. E.; Wu, Y.; Frisbie, C. D. Experimental and Theoretical Analysis of Nanotransport in Oligophenylene Dithiol Junctions as a Function of Molecular Length and Contact Work Function. ACS Nano 2015, 9, 8022-8036.

(39) Kim, B.; Choi, S. H.; Zhu, X.-Y.; Frisbie, C. D. Molecular Tunnel Junctions Based on П-Conjugated Oligoacene Thiols and Dithiols Between $\mathrm{Ag}, \mathrm{Au}$, and Pt Contacts: Effect of Surface Linking Group and Metal Work Function. J. Am. Chem. Soc. 2011, 133, 19864-19877.

(40) Slowinski, K.; Chamberlain, R. V.; Miller, C. J.; Majda, M. Through-Bond and Chain-To-Chain Coupling. Two Pathways in Electron Tunneling Through Liquid Alkanethiol Monolayers on Mercury Electrodes. J. Am. Chem. Soc. 1997, 119, 11910-11919.

(41) Love, J. C.; Estroff, L. A.; Kriebel, J. K.; Nuzzo, R. G.; Whitesides, G. M. Self-Assembled Monolayers of Thiolates on Metals as a Form of Nanotechnology. Chem. Rev. 2005, 105, 1103-1169.

(42) Jiang, L.; Sangeeth, C. S. S.; Wan, A.; Vilan, A.; Nijhuis, C. A. Defect Scaling With Contact Area in EGaIn-Based Junctions: Impact on Quality, Joule Heating, and Apparent Injection Current. J. Phys. Chem. C 2015, 119, 960-969.

(43) Jiang, L.; Sangeeth, C. S. S.; Yuan, L.; Thompson, D.; Nijhuis, C. A. One-Nanometer Thin Monolayers Remove the Deleterious Effect of Substrate Defects in Molecular Tunnel Junctions. Nano Lett. 2015, 15, 6643-6649.

(44) Slowinski, K.; Majda, M. Mercury-Mercury Tunneling Junctions - Part II. Structure and Stability of Symmetric Alkanethiolate Bilayers and Their Effect on the Rate of Electron Tunneling. J. Electroanal. Chem. 2000, 491, 139-147.

(45) Nijhuis, C. A.; Reus, W. F.; Siegel, A. C.; Whitesides, G. M. A Molecular Half-Wave Rectifier. J. Am. Chem. Soc. 2011, 133, 1539715411.

(46) Nijhuis, C. A.; Reus, W. F.; Whitesides, G. M. Molecular Rectification in Metal-SAM-Metal Oxide-Metal Junctions. J. Am. Chem. Soc. 2009, 131, 17814-17827.
(47) Yuan, L.; Breuer, R.; Jiang, L.; Schmittel, M.; Nijhuis, C. A. A Molecular Diode With a Statistically Robust Rectification Ratio of Three Orders of Magnitude. Nano Lett. 2015, 15, 5506-5512.

(48) Yoon, H. J.; Liao, K. C.; Lockett, M. R.; Kwok, S. W.; Baghbanzadeh, M.; Whitesides, G. M. Rectification in Tunneling Junctions: 2,2'-Bipyridyl-Terminated N-Alkanethiolates. J. Am. Chem. Soc. 2014, 136, 17155-17162.

(49) Kong, G. D.; Kim, M.; Cho, S. J.; Yoon, H. J. Gradients of Rectification: Tuning Molecular Electronic Devices by the Controlled Use of Different-Sized Diluents in Heterogeneous Self-Assembled Monolayers. Angew. Chem., Int. Ed. 2016, 55, 10307-10311.

(50) Qiu, L.; Zhang, Y.; Krijger, T. L.; Qiu, X.; van't Hof, P.; Hummelen, J. C.; Chiechi, R. Rectification of Current Responds to Incorporation of Fullerenes Into Mixed-Monolayers of Alkanethiolates in Tunneling Junctions. Chem. Sci. 2017, 8, 2365-2372. 